\title{
Pflichtverletzung bei gemeinschaftlicher Tatbegehung: Semantische Probleme der Beteiligungslehre
}

\author{
Urs Kindhäuser
}

\section{Problemstellung}

\47 RStGB lautete: „Wenn mehrere eine strafbare Handlung gemeinschaftlich ausführen, so wird jeder als Täter bestraft.“

Interessant an dieser Vorschrift ist zunächst ihre systematische Stellung: Mit ihr begann der Abschnitt über die Teilnahme im RStGB. Der Gesetzgeber griff damit auf die gemeinrechtliche Doktrin zurück, neben Anstiftung und Beihilfe die - im preußischen Strafrecht nicht genannte - Mittäterschaft als dritte Form der Teilnahme zu stellen. ${ }^{1}$ Kriterium der als Mittäterschaft bezeichneten Teilnahme ist die Gemeinschaftlichkeit der Tatausführung, also der Idee nach eine qualifizierte Beihilfe. Während der die Haupttat durch intellektuelle Beeinflussung veranlassende Teilnehmer unter der Bezeichnung „Anstifter“ dem ausführenden Akteur im Strafrahmen gleichgestellt wird, wird bei der physischen Teilnahme differenziert: Erschöpft sich die Hilfe in der Unterstützung einer Tatbestandsverwirklichung durch einen anderen, so ist eine Beihilfe im technischen Sinne gegeben. Wenn dagegen durch wechselseitige Hilfe ein tatbestandsverwirklichendes Geschehen gemeinsam realisiert wird, dann wird der jeweilige Tatbeitrag unter der Bezeichnung „Mittäterschaft“ (oder Miturheberschaft) der Alleintäterschaft gleichgestellt.

Da für alle Beteiligungsformen mit Ausnahme der Beihilfe im technischen Sinne derselbe Strafrahmen vorgesehen ist, erscheint die Klassifikation in Täterschaft, Mittäterschaft und Anstiftung auf den ersten Blick nur als formale Nomenklatur ohne qualitative Differenzierung. Dementsprechend charakterisiert auch $v$. Liszt in der ersten Auflage seines Lehrbuchs die Mittäterschaft allein durch das formale Kriterium der Verwirklichung wenigstens eines Tatbestandsmerkmals. Er verdeutlicht dies anhand der insoweit als anschauliche Beispiele dienenden zweiaktigen Delikte, bei de-

1 Näher hierzu Hälschner, Das gemeine deutsche Strafrecht, Bd. 1, Die allgemeinen strafrechtlichen Lehren, 1881, S. $418 \mathrm{ff}$. 
nen der eine Beteiligte den einen Akt, der andere den anderen Akt verwirklicht. $^{2}$

Bei genauerer Analyse zeigt sich jedoch, dass diese formale Klassifikation nach $v$. Liszts eigenen Prämissen nicht gelingen kann. Denn $v$. Liszt sieht in der Abgrenzung von Täterschaft und Teilnahme eine Konsequenz der Lehre vom Regressverbot: Vorsätzliche Handlungen können nach seiner Lehre nur bedingt, aber nicht verursacht sein, so dass der Teilnehmer keine Ursache zur Tatbestandsverwirklichung setzt und damit nicht selbst Täter sein kann. Da folglich bei der arbeitsteiligen Verwirklichung zweiaktiger Delikte durch zwei Beteiligte der jeweils vorsätzliche Teilakt eines Beteiligten die Ursächlichkeit des je anderen an diesem Teilakt hindert, gerät man in eine Sackgasse: Wenn jeder der Beteiligten am Teilakt des je anderen nur Teilnehmer ist, kommt es zu keiner einheitlichen Tatbestandsverwirklichung, die jeder der Beteiligten vollumfänglich als Täter zu verantworten hätte. Schlimmer noch: Es lässt sich noch nicht einmal jedem Beteiligten der von ihm verwirklichte Teilakt als Versuch der Gesamttat zurechnen, weil ja für jeden Beteiligten schon ex ante feststeht, dass diese gar nicht zur Vollendung kommen kann.

Es erscheint überflüssig darzulegen, dass sich das geschilderte Problem weder durch den subjektiven Ansatz der tradierten Rechtsprechung noch durch die Kriterien der heute vorherrschenden Tatherrschaftslehre beheben lässt. Allein dadurch, dass der eine Beteiligte ein Eigeninteresse an der Verwirklichung eines deliktischen Teilakts durch den anderen Beteiligten hat, kann er entgegen der subjektiven Lehre nicht zum Täter dieses Teilakts werden. ${ }^{3}$ Der Wille kann als handlungswirksamer Faktor nur das eigene Verhalten gestalten; auf fremdes Handeln gerichtet, erschöpft er sich in bloßem Wünschen. Und die Aufgabenverteilung bei der Ausführung eines Deliktes im Sinne der Tatherrschaftslehre erlaubt nur nach Maßgabe von Strafwürdigkeitskriterien Differenzierungen bei der Beteiligung: Der Zentralgestalt ist mehr anzulasten als der Randfigur. ${ }^{4}$ Schöne Bilder sind kein Ersatz für eine dogmatisch valide Begründung des Umschlags von Quantität in Qualität. Ohnehin lässt sich eine den Gesamtbereich des Strafrechts

2 v. Liszt, Das Deutsche Reichsstrafrecht, 1881, S. 151.

3 Grundlegend hierzu $v$. Buri, Zur Lehre von der Teilnahme an dem Verbrechen und der Begünstigung, 1860; ders., GA 1869, 234; seit RGSt 3, 181 (182 f.) ständige Rechtsprechung; umf. Darstellung und Kritik bei Birkmeyer, Die Lehre von der Teilnahme und die Rechtsprechung des Deutschen Reichsgerichts, 1890, S. 16 ff.

4 Vgl. hierzu nur Roxin, Strafrecht, Allgemeiner Teil, Bd. 2, 2003, $\$ 25$ Rn. 10 ff., 27 ff. und passim m. umf. Nachw.; zur Kritik einer solchen Bildhaftigkeit Lampe, ZStW 119 (2007), 471 (481 m. Fn. 45). 
betreffende Abgrenzung von Täterschaft und Teilnahme schon deshalb nicht mit Hilfe faktischer Kriterien wie Wille oder Tatgestaltung vornehmen, weil Täterschaft bereichsweise an persönliche Voraussetzungen gebunden ist. ${ }^{5}$

Weder die subjektive noch die materiell-objektive Beteiligungslehre sind damit - wissenschaftstheoretisch gesehen - „Theorien“. Ihre Kriterien lassen sich nicht auf einen Oberbegriff mit Erklärungskraft zurückführen, sondern sind Additionen von disparat ausgestalteten Fallgruppen. Da die Addition von Fallgruppen zu keiner inneren Geschlossenheit führt, sondern beliebig erweitert oder reduziert werden kann, taugt sie nicht zu abschließenden qualitativen Klassifikationen. So lässt sich vor allem bei der Tatherrschaftslehre deren Aufteilung der Beteiligungsformen nie falsifizieren, weil sie auf keinem durchgängig notwendigen Merkmal für Täterschaft aufbaut.

Eine Beteiligungslehre, die eine theoretische Verankerung anstrebt, muss daher zunächst den Begriff des Täters einheitlich und verbindlich für die allgemeine Straftatlehre festlegen. Durch welche Merkmale sich ein solcher Begriff definieren ließe, soll im Folgenden auf einer normtheoretischen Basis skizziert werden. Aufgrund dieser Überlegungen lassen sich sodann die spezifischen Schwierigkeiten bei der Bestimmung der Mittäterschaft im Verhältnis zur Einzeltäterschaft einerseits und zur Beihilfe andererseits aufzeigen.

\section{Täterschaft als Pflichtverletzung}

\section{Die Perspektiven ex ante und ex post factum}

Begonnen sei mit der Frage, wie sich Täterschaft im Strafrecht definieren lässt, und die normtheoretische Antwort kann nur lauten: Täter ist, wer die Pflicht verletzt, die sich aus der einem Delikt zugrundeliegenden primären Verhaltensnorm ergibt. Kurz: Täterschaft ist Pflichtverletzung.

Ein erster Schritt auf dem Weg zur Begründung dieser These ist die Unterscheidung zwischen präskriptiven und askriptiven Regeln sowie zwischen der ex ante- und der ex post-Perspektive. Die Anwendung askriptiver Regeln erfolgt ausschließlich ex post; es geht hierbei um die Zuschreibung von Verantwortung für ein Verhalten samt Folgen unter einer bestimmten

5 Daher die Begründung von Täterschaft nach dem Sonderpflichtkriterium, vgl. nur Roxin (Fn. 4), \$25 Rn. 267 ff. m. umf. Nachw. 
Beschreibung. Nach H.L.A. Harts Analyse impliziert etwa der Satz „X hat Y getötet", dass X für den Tod des $\mathrm{Y}$ verantwortlich ist, allerdings in widerlegbarer Weise. ${ }^{6}$ Das heißt: Das Zurechnungsurteil beruht auf Annahmen, die argumentativ widerlegt werden können. Im Strafrecht wird der Gegenstand der Zurechnung durch die Merkmale der Deliktstatbestände festgelegt. Kriterium der Zurechnung ist dagegen nach Maßgabe des Schuldprinzips die Vermeidbarkeit des tatbestandsverwirklichenden Geschehens bei Aufbietung erwartbarer Rechtstreue. Das Kriterium der Vermeidbarkeit verweist auf den Zeitpunkt ante factum, also auf den Zeitpunkt, zu dem sich das Subjekt der späteren Zurechnung zu einer rechtmäßigen Alternative seines tatsächlichen Verhaltens hätte entscheiden können, und zwar mit der Folge, dass die Tatbestandsverwirklichung ausgeblieben wäre.

Es liegt auf der Hand, dass das Gewicht des strafrechtlichen Vorwurfs, der ex post im Wege der Zurechnung begründet wird, auch davon abhängt, in welchem Maße das Subjekt der Zurechnung für fähig gehalten wird, sich rechtmäßig zu verhalten. Wer weiß, was er tut und dessen Bewertung kennt, wer also vorsätzlich und mit Unrechtsbewusstsein handelt, den trifft der Vorwurf mangelnder Rechtstreue härter als denjenigen, der erst bei Aufbietung verkehrsüblicher Sorgfalt die rechtlich negativen Folgen seines Verhaltens hätte abschätzen können. Auch kann ein Verhalten, je nach dem verwirklichten Unrecht, überhaupt erst dann als strafwürdig angesehen werden, wenn der Handelnde nicht nur für fähig gehalten wird, eine rechtmäßige Verhaltensalternative zu ergreifen, sondern das deliktische Geschehen sogar absichtlich oder mit sicherem Wissen herbeiführte. Alles dies sind sanktionsrelevante Kriterien der Zurechnung, durch die ex post der Schuldvorwurf gegenüber dem Handelnden konstituiert wird.

Während sich die ex post-Perspektive auf dasjenige bezieht, was getan wurde und wie dies zu bewerten ist, betrifft die ex ante-Perspektive die Frage, was getan werden kann und soll. Aus der Sicht ex ante geht es um die Bestimmung der nach Maßgabe rechtlicher Präferenzen richtigen Entscheidung unter Berücksichtigung der dem Handelnden situativ zur Verfügung stehenden Kenntnisse, Fähigkeiten und Verhaltensmöglichkeiten. Diese Kriterien der ex ante erforderlichen Fähigkeit zum Erkennen und Umsetzen des rechtlich richtigen Verhaltens dienen ex post als Kriterien der Zurechnung. Für das ex ante Vermeidbare, aber nicht Vermiedene wird das Subjekt der Zurechnung ex post zur Verantwortung gezogen. Insoweit bedarf es zur Bestimmung täterschaftlicher Verantwortung einer

6 Grundlegend Hart, The Ascription of Responsibility and Rights, Proceedings of the Aristotelian Society, 49 (1948/49), S. $171 \mathrm{ff}$. 
Analyse der Entscheidungssituation, in der sich das spätere Subjekt der Zurechnung ante factum befand.

\section{Verhaltensnormen}

Betrachten wir zunächst das Sollen, an dem sich die ex ante-Entscheidung auszurichten hat, und zwar in der Form präskriptiver Präferenzen, also in der Form von Verhaltensnormen. Der Einfachheit halber seien nur Gebote und Verbote in den Blick genommen, die sich im Strafrecht zwanglos aus den Tatbestandsformulierungen ableiten lassen, genauer: aus den Grundtatbeständen, da alle weiteren Merkmale nur ex post anzuwendende Strafzumessungskriterien sind. ${ }^{7}$ In ihrer präskriptiven Funktion sind Normen Verhaltensanweisungen, die sich mit Hilfe deontischer Operatoren wie „sollen“ oder „dürfen“ ausdrücken lassen, etwa: „Du sollst keine fremde Sache beschädigen“.

Auf Präskriptionen lassen sich die Regeln der deskriptiven Logik nicht (unmittelbar) anwenden, da logische Folgerungen wahrheitsbewahrende Relationen sind, Präskriptionen aber nicht wahr oder falsch sein können. ${ }^{8}$ Allerdings haben Normen einen propositionalen Gehalt, der sich isoliert vom präskriptiven Operator in der Normformulierung auch deskriptiv verwenden lässt. Im Beispielsfall könnte das Verbot der Sachbeschädigung durch eine Trennung des die präskriptive Funktion ausdrückenden Operators „gesollt" von der Normproposition „keine fremde Sache beschädigen“ sprachlich gefasst werden, etwa: „gesollt: keine fremde Sache beschädigen“.

Damit stellt sich das Problem, wie Normen befolgt werden. Denn das Verhalten, durch das normgemäß gehandelt wird, ist samt den hierzu erforderlichen physischen, intellektuellen und volitiven Voraussetzungen faktischer Natur und damit Gegenstand deskriptiver Aussagen. Ein in der heutigen Strafrechtsdogmatik häufig anzutreffender Fehler besteht darin, die Bedingungen der Normbefolgung als Merkmale der Normproposition

7 Beispielhaft: Eine gefährliche Körperverletzung ist sub specie Verhaltensnorm nicht „verbotener“ als eine einfache Körperverletzung, wohl aber ist sie nach Maßgabe der Strafwürdigkeit gravierenderes Unrecht als eine leichte Verletzung.

8 Auf die Streitfrage, ob es einer besonderen Logik der Normen (in präskriptiver Funktion) bedarf, kann und soll hier nicht näher eingegangen werden, sie ist im hiesigen Kontext unerheblich; vgl. zur Problematik nur Rödig, Schriften zur juristischen Logik, 1980, S. 169 ff., 185 ff.; Weinberger, Rechtslogik, 2. Aufl. 1989; $v$. Wright, Normen, Werte, Handlungen, 1994, S. $56 \mathrm{ff}$. 
anzusehen. Insbesondere die finale Lehre hat aus der richtigen Einsicht, dass Normen nur durch intentionales Handeln befolgt werden können, den Fehlschluss gezogen, dass Normen intentionales Handeln vorschrieben oder untersagten. ${ }^{9}$ Doch wäre Inhalt einer Norm ein intentionales Handeln, so müsste derjenige, der eine solche Norm befolgen wollte, eine entsprechende Intention haben wollen.

Im Beispiel der Sachbeschädigung lautet die Präskription „Du sollst keine fremde Sache beschädigen“ und nicht etwa „Du sollst nicht die Absicht haben, eine fremde Sache zu beschädigen." Verboten ist das Beschädigen, nicht die Absicht zu beschädigen. Dementsprechend soll eine Person, die das Verbot befolgen will, keine fremde Sache beschädigen, und nicht etwa nicht die Absicht haben, eine fremde Sache zu beschädigen. Norminhalt und Normbefolgung sind zweierlei, so dass auch die faktischen Voraussetzungen der Normbefolgung nicht zum Inhalt der Normproposition gehören können, was im Übrigen auch gegen das logische Verbot der Selbstbezüglichkeit verstieße. ${ }^{10}$

\section{Norm und Pflicht}

Wie also werden Normen befolgt? Im Strafrecht stellt sich mit Blick auf das Befolgen von Ge- und Verboten ein schwieriges Problem. Das strafrechtliche Garantieprinzip (Art. 103 Abs. 2 GG) verlangt, dass nur dasjenige Verhalten samt Folgen Gegenstand eines Unrechtsvorwurfs sein darf, das exakt unter die Beschreibung eines Deliktstatbestandes fällt. Dementsprechend kann auch nur die tatbestandliche Verhaltensbeschreibung samt Folgen Proposition der sanktionierten Verhaltensnorm sein, was zu sehr engen Normformulierungen führt. Aus dem Delikt der Sachbeschädigung lässt sich folglich nur die Normproposition logisch ableiten: „beschädigen einer fremden Sache“. Unter diese Beschreibung fällt aber ein Verhalten erst in dem Zeitpunkt, in dem eine Sache beschädigt ist. Die Handlung der Sachbeschädigung lässt sich begrifflich nur ex post zurechnen. ${ }^{11}$

Aus dieser begrenzten begrifflichen Reichweite der Verhaltensnorm folgt, dass sich die Beschreibung der Verhaltensalternative, die der Täter

9 Beispielhaft Armin Kaufmann, Lebendiges und Totes in Bindings Normentheorie, 1954, S. 102, 106 und passim.

10 Vgl. hierzu nur Vogel, Norm und Pflicht bei unechten Unterlassungsdelikten, 1993, S. 41 f. m.w.N.

11 Grundlegend zur Beschreibungsabhängigkeit von Handlungen Anscombe, Intention, 1957, S. $37 \mathrm{ff}$; $v$. Wright, The Varieties of Goodness, 1963, S. $123 \mathrm{f}$. 
ergreifen soll, um eine Norm zu befolgen, nicht dieser Norm selbst begrifflich entnehmen lässt. Ein weiteres einfaches Beispiel: Ein Unglücksfall hat sich ereignet, und eine anwesende Person soll dem Gebot der Hilfeleistung in Unglücksfällen nachkommen. Es gibt keine strafrechtliche Verhaltensnorm des Inhalts, die ihrem Adressaten vorschreibt, zu einem Ertrinkenden zu schwimmen und ihn aus dem Wasser zu ziehen. Die „Norm“, so handeln zu sollen - sie sei einmal „ $m$ " genannt -, ist in dem Gebot, bei Unglücksfällen Hilfe zu leisten - hier „,“ genannt -, ersichtlich nicht begrifflich erhalten. Was also ist „ $m$ “?

Dass diese Frage - in dieser Formulierung - in der gängigen Strafrechtsdogmatik nicht behandelt wird, mag mit der Offenkundigkeit zusammenhängen, dass der Gebotsadressat nach Maßgabe von " $m$ “ handeln muss, um das Gebot "s“ zu erfüllen. Deshalb mag es auch auf der Hand liegen, dass „ $m^{\prime \prime}$ genau das ist, was die Gebotsnorm „s“ von ihrem Adressaten hier verlangt. Dies könnte zu der Annahme verleiten, dass „ $m^{\prime \prime}$ selbst die Gebotsnorm sei. Nur kann dies, nimmt man das strafrechtliche Garantieprinzip ernst, nicht die Lösung des Problems sein. Der Interpret darf nicht kurzerhand den Text des zu Interpretierenden nach eigenem Gusto abändern oder völlig neu formulieren.

Festzuhalten ist also, dass ,,$"$ und,$m$ " zwei verschiedene Normen sind und dass, bei genauerem Hinsehen, „ $m$ " auch ein anderer Normtyp ist als „“. Das Hilfeleistungsgebot „s“ drückt gewissermaßen das Ziel aus, das erreicht werden soll, während die Verhaltensanweisung " $m$ “ - zumindest ein rationales Mittel nennt, um das Ziel zu erreichen. Es erscheint deshalb auch angemessener, bei der Formulierung von " $m^{\prime \prime}$ das deontische Verb „sollen“ durch das Verb „müssen“ zu ersetzen. Es gibt keine strafbewehrte Norm, die dem Retter vorschreibt, er solle schwimmen. Denn das Recht verlangt von ihm ja nicht das Schwimmen, sondern das Leisten von Hilfe. Das Müssen des Schwimmens folgt vielmehr aus dem gesetzten Ziel des Rettens, falls dieses in der konkreten Situation nur durch Schwimmen erreicht werden kann.

Das Müssen entspringt demnach einer rationalen Deliberation, die zunächst deskriptiver Natur ist. Angenommen, in der fraglichen Situation gäbe es kein staatliches Gebot der Hilfeleistung in Unglücksfällen. Der Retter möchte vielmehr aus freien Stücken den Ertrinkenden retten und überlegt, was er tun kann, um dieses Ziel zu erreichen. Sollte sich hierzu nur das Hinschwimmen und aus dem Wasser Ziehen des Ertrinkenden als taugliches Mittel erweisen, so muss der Retter schwimmen, wenn die Rettung sein dominantes Ziel ist. So gesehen ist „ $m$ “ also keine Präskription, sondern die Konsequenz aus einem praktischen (rationalen) Schluss zur Realisierung eines gesetzten Ziels, hier: zur Erfüllung des Gebots „s“. Oder 
anders ausdrückt: Das Müssen von „ $m^{\prime \prime}$ ist Ausdruck praktischer Notwendigkeit.

Eine Verhaltensanforderung, die auf dem Umstand beruht, dass eine bestimmte Verhaltensalternative ergriffen werden muss, um ein gesolltes Ziel zu erreichen, nennt $v$. Wright ein „technisches Sollen“. ${ }^{12}$ Diese Bezeichnung bringt den instrumentellen Charakter der Zweck-Mittel-Relation gut zum Ausdruck und damit auch die Verbindung zwischen dem Sollen des Ziels und dem Müssen des Mittels. Denn die Formulierung von „ $m$ “ ist elliptisch, genauer: sie impliziert einen unausgesprochenen hypothetischen Imperativ: Der Retter soll „ $m^{\prime}$ ausführen, weil die Norm „s“, Hilfe zu leisten, nur dann erfüllt wird, wenn „ $m^{\prime \prime}$ getan wird. Das Müssen entspringt also dem Wollen des Gesollten.

Noch eine Besonderheit von „ $m$ “ ist bemerkenswert. Während sich das Gebot der Hilfeleistung allgemein an alle Personen richtet, die Adressaten der Normen des deutschen Strafrechts sind, gilt „ $m$ “ lediglich für denjenigen Normadressaten, der sich in einer bestimmten Situation befindet und über eine bestimmte Handlungs- und Motivationsfähigkeit verfügt. „ $m$ “ ist also ein situativ konkretisiertes und hinsichtlich des erforderlichen Handelns individualisiertes „Müssen“. Könnte der Retter nicht schwimmen, „müsste“ er auch nicht schwimmen; „ $m$ “ hätte unter diesen Umständen einen anderen Inhalt (oder ließe sich - bei Handlungsunfähigkeit - gar nicht begründen). Der betreffende Normadressat „müsste“ dann ggf. andere Personen verständigen oder sich ein Boot verschaffen, um zu dem Ertrinkenden zu gelangen.

Insoweit hat hier der Satz, dass „Sollen“ ein Sein (Können) impliziert, einen Sinn. Denn das „Sollen“ von „ $m$ “ ist deskriptiv, ist also kein „echtes“ - d.h. vom Sein kategorial verschiedenes - Sollen, sondern Ausdruck eines hypothetischen Müssens, um einem kategorischen Sollen nachzukommen. Durch „ $m$ “ wird das Sollen der kategorischen Norm mit dem So-Sein einer individuellen Person in einer konkreten Situation verbunden. Oder anders formuliert: „ $m$ “ bindet einen bestimmten Normadressaten an eine Norm nach Maßgabe der ihm möglichen und von ihm erwarteten Leistungsfähigkeit. Diese Gebundenheit einer Person an eine Norm sei Pflicht genannt. In diesem Sinne können Normen als Verpflichtungsgründe für Handlungen verstanden werden. ${ }^{13}$

12 v. Wright (Fn. 8), S. 36.

13 Grundlegend hierzu Raz, Praktische Gründe und Normen, 2006, S. 33 und passim; vgl. insoweit auch Armin Kaufmann (Fn. 9), S. 129 ff. 


\section{Praktisches Schließen}

Das technische „Sollen“ der Pflicht lässt sich mit Hilfe eines praktischen Syllogismus bestimmen. ${ }^{14}$ Ein solcher Schluss ist formal dem logischen Schluss-Schema ${ }^{15}$ nachgebildet. Der praktische Syllogismus nennt in der Oberprämisse die Norm-Proposition und in der Konklusion das pflichtgemäße Verhalten. Verbunden werden Oberprämisse und Konklusion durch die für die jeweilige Handlung relevanten Kenntnisse und physischen Fähigkeiten des konkreten Normadressaten. Die praktische Notwendigkeit, das in der Konklusion genannte Handeln auszuführen, ergibt sich, wenn der in der Oberprämisse genannte deontische Operator der Norm durch einen Ausdruck für das (dominante) Wollen des Adressaten ersetzt wird. Das heißt: Aus der Norm „Es ist gesollt, bei Unglücksfällen Hilfe zu leisten“ wird die Intention „Eine (rechtstreue) Person $P$ will (unbedingt) bei Unglücksfällen Hilfe leisten“. Der praktische Schluss hat also folgende allgemeine Form:

- Oberprämisse: Eine Person $P$ will (das gebotene) $x$ tun.

- Unterprämisse: $P$ nimmt an, dass sie $x$ (nur) ausführen kann, wenn sie $y^{16}$ tut.

- Konklusion: Also muss $P$ y tun.

Bei Begehungsdelikten ist umgekehrt die Vornahme einer Handlung, die den Tatbestand verwirklicht, verboten und damit das Ergreifen irgendeiner rechtmäßigen Verhaltensalternative geboten. Als Konklusion des entsprechenden praktischen Schlusses kommt daher jedes rechtmäßige Verhalten in Betracht, das als Unterlassen des tatbestandsverwirklichenden Tuns anzusehen ist. Für einen Normadressaten, der (dominant) gewillt ist, strafrechtliche Verbote zu befolgen, hätte der praktische Schluss somit folgende Gestalt:

- Oberprämisse: Eine (rechtstreue) Person $P$ will (das verbotene) $x$ unterlassen.

- Unterprämisse: $P$ nimmt an, dass sie $x$ nur (sicher) unterlassen kann, wenn sie $y$ unterlässt.

- Konklusion: Also muss $P$ y unterlassen.

14 Näher $v$. Wright, Handlung, Norm und Intention, 1977, S. 42 ff.

15 Hierzu Essler, Einführung in die Logik, 2. Aufl. 1969, S. $41 \mathrm{ff}$.

16 Bei gleichermaßen effizienten Handlungen kann $y$ auch durch eine Disjunktion ersetzt werden (z.B. $y_{1}$ oder $y_{2}$ oder $y_{3}$ ), mit der Folge, dass von $P$ eine der disjunktiv verbundenen Handlungen ausgeführt werden „muss“. 


\section{Tat, Tatherrschaft und Täter}

Will man nun die Begriffe Tat und Täter in diese Konzeption der strafrechtlichen Normentheorie einfügen, so ergibt sich die folgende sinnvolle Möglichkeit: „Tat“ ist das unter die Beschreibung des Deliktstatbestandes fallende Verhalten samt einschlägigen Folgen und Begleitumständen, also der ex post zu konstituierende Gegenstand der Zurechnung. „Tatherrschaft" besitzt wiederum, wer die Tatbestandsverwirklichung durch pflichtgemäßes Alternativverhalten vermeiden konnte und musste. „Täter“ ist schließlich das Subjekt der Zurechnung, also derjenige, der in der tatbestandlichen Deskription des Geschehens als „wer“ bezeichnet wird. Oder anders formuliert: Täter ist, wer aufgrund einer Pflichtverletzung für die Tatbestandsverwirklichung strafrechtlich verantwortlich ist.

Bereits die Dogmatik des 19. Jahrhunderts ${ }^{17}$ durchzieht der Gedanke, dass derjenige Täter (Urheber) sei, der eine Tatsache zu verantworten hat, die zu dem gesetzlichen Begriff eines Verbrechens gehört. ${ }^{18}$ So umschreibt Adolf Merkel denjenigen als Täter, „auf welchen die gesetzliche Definition einer Deliktsart und das mit ihr verbundene Strafmaß unmittelbar Bezug haben, welcher sich also mit seinem Thun oder Lassen jener Definition unmittelbar subsumieren läßt oder, was dasselbe ist, derjenige, der die in dem betreffenden Gesetze vorausgesetzte, die gesetzlichen Deliktsmerkmale zur Erscheinung bringende Handlung, d.i. die ,Haupthandlung' oder ,Ausführungshandlung' vornimmt“ ${ }^{19}$ Zum Tatbestand gehöre u.a. auch „die Verletzung eines besonderen Pflichtverhältnisses“, so dass nur derjenige Täter sein könne, „der in diesem Pflichtverhältnis steht“ ${ }^{20}{ }^{20}$ Dagegen sei derjenige nicht Täter, „in dessen Person oder Handlung die gesetzlichen Merkmale fehlen“. ${ }^{21}$ Dies ist nicht nur, wie Roxin schreibt, „ein Gedanke

17 Umf. Nachw. bei Birkmeyer, Teilnahme am Verbrechen, VDA, Bd. 2, 1908, S. 97; ders. (Fn. 3), S. 6 f.; ferner Haas, Die Theorie der Tatherrschaft und ihre Grundlagen, 2008, S. $65 \mathrm{ff}$.

18 Vgl. Stübel, Ueber den Thatbestand der Verbrechen, die Urheber derselben und die zu einem verdammenden Endurtheile erforderliche Gewißheit des erstern, besonders in Rücksicht der Tödtung nach gemeinen in Deutschland geltenden und Chursächsischen Rechten, 1805, $\$ 29$ S. 34, ferner $\$ 23$ S. 29.

19 Merkel, Lehrbuch des Deutschen Strafrechts, 1889, S. 137 (Hervorhebungen nicht im Original); vgl. auch Berner, Lehrbuch des Deutschen Strafrechtes, 14. Aufl. 1886, S. 151.

20 Merkel (Fn. 19), S. 140.

21 Merkel (Fn. 19), S. 139 f. 
von beinahe zwingender Evidenz".22 Es ist vielmehr die dem strafrechtlichen Garantieprinzip allein entsprechende Auslegung des Gesetzeswortlauts. Ein in diesem Sinne am Gesetzeswortlaut orientiertes Verständnis der Beteiligungslehre wird seit $v$. Birkmeyer formal-objektive Theorie genannt. ${ }^{23}$

Die formal-objektive Theorie beruht auf einer mit der Äquivalenztheorie der Kausalität nicht zu vereinbarenden Voraussetzung, nämlich der Ungleichwertigkeit aller notwendigen Bedingungen. Denn die einzelnen notwendigen Bedingungen eines Erfolgs verlieren ihre Gleichwertigkeit, wenn sie aus rechtlicher Perspektive betrachtet werden. Beispielhaft: Wenn B dem A ein Messer gibt, mit dem dieser den C tödlich niedersticht, so lässt sich sagen: „A hat $C$ durch den Messerstich getötet“. Es wäre dagegen sprachlich verfehlt zu sagen: „B hat $\mathrm{C}$ getötet, indem er A das Messer gab, mit dem dieser den $C$ niederstach“. Nur das Verhalten von A lässt sich als „töten“ beschreiben. Dass in diesem Beispielsfall A und B jeweils notwendige Bedingungen für den Tod des $\mathrm{C}$ gesetzt haben, ist als Faktum ebenso richtig und zugleich strafrechtlich irrelevant wie der Umstand, dass $\mathrm{C}$, um sterben zu können, vorher lebendig gewesen sein musste. Da alle Elemente, die den Zustand der Welt zum Zeitpunkt $t$ konstituieren, notwendig für diesen Zustand der Welt und damit auch für den Tod des C zum späteren Zeitpunkt $t_{+1}$ sind, kommt die Erkenntnis, dass auch das Verhalten von A und B sowie die Existenz von C selbst notwendige Bedingungen für dessen Tod sind, der Entdeckung des Mondes gleich.

Unter die Beschreibung „töten eines anderen“ ist ausschließlich das Verhalten des A zu subsumieren. Dagegen fällt das Verhalten des B nicht unter diese Beschreibung, da er den Tod des $\mathrm{C}$ nicht dadurch, dass er dem A das Messer reichte, herbeigeführt hat. Demnach musste auch B sein Verhalten nicht nach Maßgabe des Totschlagsverbots unterlassen. Unter den gegeben kausalen Rahmenbedingungen war das Verhalten des B als solches weder hinreichend, um den Tod des $\mathrm{C}$ herbeizuführen, noch zu diesem Zeitpunkt notwendig für dieses Ereignis. Hätte A nicht zugestochen, wäre der Umstand, dass ihm B ein Messer reichte, sub specie Tötungsverbot völlig irrelevant geblieben. Zu einer notwendigen Bedingung für den Tod des $\mathrm{C}$ wurde das Verhalten des B erst vermittelt durch das Verhalten des A; erst A hat durch sein Verhalten die für den Tod des $C$ relevanten Umstän-

22 Roxin, Täterschaft und Tatherrschaft, 9. Aufl. 2017, S. 35 (Hervorhebungen nicht im Original).

23 Birkmeyer (Fn. 17), S. 21. 
de zu einer für dieses Ereignis hinreichenden Gesamtbedingung komplettiert. $^{24}$

Legt man der Allgemeinen Straftatlehre einen semantisch begründeten restriktiven Täterbegriff zugrunde - und hieran führt m.E. bei Beachtung des Garantieprinzips kein Weg vorbei -, dann sind alle Formen der Beteiligung, die über eine eigenhändige Täterschaft hinausgehen, Ausweitungen der strafrechtlichen Verantwortlichkeit. Alle die Strafbarkeit ausweitenden Beteiligungsformen bedürfen damit einer konstitutiven gesetzlichen Regelung und implizieren jeweils spezifische Normen. Im Anschluss an Adolf Merkel $^{25}$ kann man die Teilnahmehandlungen des Anstifters und Gehilfen als tatbestandlich umschriebene „Hilfshandlungen“ zur Durchführung der „Haupthandlung" des Täters bezeichnen und aus ihnen entsprechende sekundäre Verhaltensnormen ableiten.

\section{Mittäterschaft}

\section{Grenzen der Vermeidbarkeit}

Auch mittelbare Täterschaft und Mittäterschaft sind gegenüber der eigenhändigen Alleintäterschaft Ausdehnungen strafbaren Verhaltens, die in $\$ 25$ Abs. 1 Alt. 2 und Abs. 2 StGB ihre gesetzlichen Grundlagen haben. Bei der Mittäterschaft, um die es nunmehr ausschließlich gehen soll, ist entgegen den Prämissen der materiell-objektiven Beteiligungslehre schon aufgrund der Tatbestandsfassungen des BT klar, dass die dortigen Verhaltensbeschreibungen nicht stets auf alle Beteiligten gleichermaßen zutreffen können. Begehen X und Y einen Raub in der Weise, dass X das Opfer festhält und Y dessen Brieftasche wegnimmt, dann passt die deliktische Tatbestandsbeschreibung auf keine der beiden Verhaltensweisen in vollem Umfang.

In solchen Konstellationen kann folglich von keinem der potenziell als Mittäter anzusehenden Beteiligten die rechtswidrige Gesamttat allein durch eigenes pflichtgemäßes Verhalten vermieden werden. Dies ist insoweit eine Binse, als Handlungsalternativen stets nur Alternativen des eige-

24 Im Alltag bezeichnet man gewöhnlich nur solche Verhaltensweisen als „verursachend", die einen Erfolg ceteris paribus hinreichend bedingen, indem sie zu einem bereits gegebenen Komplex von Umständen komplettierend hinzutreten, näher hierzu Kindhäuser, ZIS 2016, $574 \mathrm{ff}$.

$25 \operatorname{Merkel~(Fn.~19);~vgl.~auch~Hälschner~(Fn.~1),~S.~} 418$ ff. 
nen Verhaltensspielraums sein können. Man kann es zwar unterlassen, Geige zu spielen, indem man ein Buch liest, aber man kann es nicht unterlassen, dass eine andere Person Geige spielt. Man kann allenfalls eine andere Person daran hindern, etwas zu tun, etwa Geige zu spielen; eine ersichtlich ganz andere Fallgestaltung. Im Falle des Raubes kann daher X nur das Festhalten und Y nur die Wegnahme unterlassen. Dies wäre auch nicht weiter erwähnenswert, wenn nicht - nach gängiger Doktrin im Strafrecht - sowohl X als auch Y jeweils die Gesamttat als je eigenes Unrecht zugerechnet würde, also X und Y so gestellt würden, als hätte jeder von ihnen den Raub allein begangen. Kann jedoch - mit Blick auf das Schuldprinzip - einem Beteiligten die Verantwortlichkeit für eine Tat zugeschrieben werden, die er durch eigenes pflichtgemäßes Alternativverhalten nicht vollumfänglich vermeiden konnte?

Während niemand auf den Gedanken käme, einer Einzelperson ein tatbestandsverwirklichendes Verhalten, zu dem sie keine Alternative hatte, als Pflichtverletzung zuzurechnen, soll - so etwa die Tatherrschaftslehre schon ein arbeitsteiliges Vorgehen ausreichen, um Verantwortung für die Gesamttat zu tragen. Doch wie der Ausdruck Arbeitsteilung besagt, erbringt ein Mitarbeiter in der alltäglichen Arbeitswelt nur eine Teilleistung und kann folglich auch nicht den gesamten Gewinn einstreichen bzw. muss nicht den gesamten Verlust tragen.

\section{Grenzen individueller Verantwortlichkeit}

Angesichts der Unmöglichkeit, die Verwirklichung eines Deliktes durch tatbestandliche Teilakte mehrerer Beteiligter als die "natürliche“ Handlung nur eines der Beteiligten zu rekonstruieren, liegt es nahe, die einzelnen Teilakte als Leistung einer Kollektivperson zu betrachten. Die Zugehörigkeit zu einem solchen Kollektiv soll sodann der Grund sein, jedem der Beteiligten die Gesamttat als eigene Tat zuzurechnen.

Nun ist die Charakterisierung einer mittäterschaftlichen Tatbestandsverwirklichung als Leistung einer Kollektivperson lediglich eine Bezeichnung, keine Begründung. Wie es möglich sein soll, Verantwortung durch die Einführung eines Gesamtsubjekts zunächst zu konstituieren und sodann auf jedes seiner (unselbständigen) Glieder zu übertragen, bleibt ein Rätsel. Jedenfalls hat nach den Wertungen der Alltagsintuition die Leistung eines Kollektivs (nur) eine kollektive Verantwortung als Kehrseite. Sachgerecht erscheint so zwar die Zuschreibung von Verantwortung für die Beteiligung an der Kollektivleistung, kaum aber die Gleichsetzung der einzelnen Beteiligung am Kollektiv mit der Tat des Kollektivs insgesamt. 
Doch selbst wenn die Konstruktion eines Gesamtsubjekts, dessen Handeln jedem der Beteiligten aufgrund objektiv passender Beiträge als jeweils eigenes Handeln zuzurechnen ist, plausibel wäre, stellte sich das Problem, warum - auf das Strafrecht bezogen - nur Mittäterschaft nach diesem Modell zu begründen sein sollte. Wäre das Modell einer Kollektivperson tragfähig, müsste vielmehr auch der Teilnehmer für die dem Kollektiv zugeschriebene Tat als eigene haften. Dass aber Täter und Teilnehmer nicht als Glieder einer Kollektivperson gedeutet werden können, geht schon aus den Teilnahmevorschriften ( $\$ \$ 26 \mathrm{f}$. StGB) selbst hervor; diese sprechen von der Teilnahme an der rechtswidrigen Tat eines anderen. Ferner stehen der Annahme eines von einem Gesamtsubjekt begründeten Unrechts die echten Sonderdelikte entgegen: Der Extraneus, der auf Geheiß eines Sonderpflichtigen eigenhändig den Tatbestand eines echten Amtsdeliktes verwirklicht, haftet per se nicht als Täter, so dass auch ein entscheidender Beitrag zum Gelingen der Tat noch nicht deren Zurechnung als eigene Handlung zu begründen vermag.

Joerden, ein Befürworter der Konstruktion, Mittäterschaft als Handeln einer Kollektivperson zu begreifen, räumt ein, dass ein logischer Schluss von der Verantwortlichkeit des Kollektivs auf die volle Verantwortung auch jedes einzelnen Mitglieds nicht möglich sei; es bleibe hier bei einem Postulat. ${ }^{26}$ Soweit Kollektive im rechtsgeschäftlichen Bereich mit Handlungsfähigkeit ausgestattet werden, sind sie selbst - mehr oder weniger umfassend - Träger von Rechten und Pflichten und dienen damit der Haftungsbegrenzung der für sie handelnden Personen. Jedoch dürfte der umgekehrte Weg, die Haftung des einzelnen aus der von ihm gar nicht vollumfänglich vermeidbaren Gesamttat abzuleiten, jedenfalls für das Strafrecht versperrt sein. Hier geht es um die Begründung von Verantwortlichkeit kraft individueller Vermeidbarkeit; jeder Beteiligte soll nach Maßgabe seiner Schuld bestraft werden $(\$ 29$ StGB). Wenn sich, wie Joerden zutreffend sagt, von der Tat eines Kollektivs nicht auf die strafrechtliche Verantwortlichkeit des einzelnen Mitglieds für diese Tat logisch schließen lässt, dann ist eine solche Konstruktion mangels logischer Schlüssigkeit auch zur Begründung von Mittäterschaft nicht brauchbar.

Wechselt man von der ex post-Perspektive der Zurechnung zur ex antePerspektive, stellt sich das weitere Problem, wie ein Kollektiv als Kollektiv soll Normen befolgen können. Man wird, wie auch immer man die Dinge dreht und wendet, nicht umhinkönnen, letztlich den einzelnen Beteiligten

26 Joerden, Strukturen des strafrechtlichen Verantwortlichkeitsbegriffs: Relationen und ihre Verkettungen, 1988, S. 79 f. 
als Normadressaten anzusehen. Und damit sind die Pflichtenstellungen der einzelnen wieder parzelliert.

\section{Wechselseitige Repräsentation}

Daraus folgt: Mittäterschaftliches Unrecht ist wie das Unrecht der Teilnahme als akzessorisches Unrecht zu begreifen; das selbst zu verantwortende Unrecht ist abhängig von dem durch eine andere Person begangenen Unrecht. Das heißt: Der Mittäter wird neben dem eigenen Verhalten auch für etwas verantwortlich gemacht, was eine andere Person begangen hat. Insoweit ist die Mittäterschaft ganz im Sinne der früheren Rechtslage eine Form der Teilnahme, die sich nicht im intellektuellen oder physischen Beitrag zur Tat eines anderen erschöpft, sondern durch eine gemeinschaftliche Vorgehensweise qualifiziert wird. ${ }^{27}$ Gemeinschaftlichkeit ließe sich insoweit definieren als wechselseitige Teilnahme.

In diesem Sinne wäre es in sich schlüssig, gemäß der formalen Systematik $v$. Liszts für die mittäterschaftliche Beteiligung die Verwirklichung eines Tatbestandsmerkmals zu verlangen. Mittäter wäre dann, wer zugleich für sich und für den anderen durch die Verwirklichung zumindest eines Tatbestandsmerkmals zur Realisierung des Unrechts einer Gesamttat beiträgt. Diese Konzeption impliziert eine Form der Zurechnung, die dem Recht durchaus geläufig ist, nämlich die Stellvertretung. Hier liegt m.E. auch der durchaus sinnvolle Kern der Tatherrschaftslehre: Repräsentant einer gemeinschaftlichen Tatbegehung kann nur sein, wer eine repräsentative Rolle spielt, namentlich im Rahmen einer funktionalen Arbeitsteilung beteiligt ist. ${ }^{28}$

Die heute vorherrschende Doktrin verlangt für mittäterschaftliche Haftung jedoch keine zumindest anteilige Tatbestandsverwirklichung. Dagegen ist nichts einzuwenden, wenn man Mittäterschaft als qualifizierte Form der Teilnahme und damit als Kriterium der Zurechnung von unrechtmäßigem Drittverhalten begreift. Unter Strafwürdigkeitsgesichts-

27 Vgl. auch Hälschner (Fn. 1), S. 420: „eigenthümliche Gestaltung der Theilnahme“.

28 Vgl. auch Binding, Strafrechtliche und strafprozessuale Abhandlungen, Bd. 1, 1915, S. 298; Haas, Die Theorie der Tatherrschaft und ihre Grundlagen, 2008, S. 112 ff.; Kindhäuser, in: Hollerbach-FS, 2001, S. 627 ff. Diesem Ansatz liegt der auf das Römische Recht zurückgehende gemeinrechtliche Grundsatz „quod quis per alium facit, per se ipsum facere videtur“ zugrunde, hierzu Carpzov, Practica nova imperialis Saxonica rerum criminalium in partes III, 1635, pars I, quaestio IV, 11. 
punkten mag auch eine besonders gewichtige Beteiligung an einer Gesamttat dem Unrecht der Tat eines Einzeltäters gleichzustellen sein, ja dieses fallweise sogar übersteigen. Es ist ja zu bedenken, dass die Beteiligung mehrerer schnell das Qualifikationsmerkmal der Bande erfüllen kann. Der Verzicht auf ein formales Kriterium im Sinne $v$. Liszts hat freilich auch zur Folge, dass die für das Strafmaß bedeutsame Abgrenzung zwischen Mittäterschaft und Beihilfe verwischt wird, ein für ein rechtsstaatlich liberales Strafrecht, wie es $v$. Liszt vorschwebte, nicht gerade attraktiver Gedanke.

Zurechnungsgrund für das Unrecht der Gesamttat kann nicht, um es zu wiederholen, der Vorwurf an den einzelnen Mittäter sein, er habe durch eigenes pflichtgemäßes Verhalten die Realisierung der Gesamttat vermeiden können. Daraus folgt, bezogen auf den Zeitpunkt ex ante, dass die vom Mittäter zu befolgende Norm nicht mit der Norm identisch sein kann, die der Einzeltäter pflichtwidrig verletzt. Vielmehr muss die Norm, die sich an potenzielle Mittäter als Adressaten richtet, wie dies auch bei den anderen Teilnahmeformen der Fall ist, eine Bezugnahme auf das Verhalten der je anderen Beteiligten implizieren, und zwar hier durch das Merkmal der Gemeinschaftlichkeit. Die Norm des Raubes kann bei mittäterschaftlicher Begehungsweise also nicht lauten: „es ist verboten, mit Gewalt gegen eine Person ... wegzunehmen“, sondern: „es ist verboten, zu mehreren gemeinschaftlich mit Gewalt gegen eine Person ... wegzunehmen".

Die normtheoretische Analyse mittäterschaftlicher Tatbegehung ist damit keineswegs beendet, im Gegenteil: Es ist noch die schwierige Frage zu beantworten, wie sich die Pflichten mehrerer Personen mit Hilfe des praktischen Syllogismus erfassen lassen. Schwierigkeiten dürften hierbei weniger die Begehungs- als vielmehr die Unterlassungsdelikte bereiten. Ein einfaches Beispiel: Eine Person droht zu ertrinken, von mehreren Anwesenden ist jedoch keiner in der Lage, allein rettend einzugreifen. Der gängige Ausweg, bei Unterlassungen jeden Garanten zum Alleintäter zu erklären, dürfte in diesem Fall versperrt sein. Hier ist demnach noch viel Arbeit zu bewältigen. Eine Arbeit, von der allerdings zu befürchten ist, dass sie nicht gern geleistet wird. Denn wie die vorangegangenen Ausführungen gezeigt haben mögen, verlangt ein analytisches Vorgehen zwar einen erheblichen Aufwand an theoretischer Begrifflichkeit, führt aber in der Regel nur zu trivialen Ergebnissen. Das Kaninchen, das der Analytiker mehr oder weniger elegant aus dem Zylinder holt, muss eben vorher schon, wenn auch verborgen, darin gewesen sein. 